\title{
Comparison of Cold Responses for Orthologs of Cabbage Vernalization- related Genes
}

\author{
Etsuko Itabashi $^{1 *}$, Daniel J. Shea ${ }^{2}$, Nobuko Fukino ${ }^{1}$, Ryo Fujimoto ${ }^{3}$, Keiichi Okazaki², \\ Tomohiro Kakizaki ${ }^{1}$ and Takayoshi Ohara ${ }^{1}$
}

${ }^{1}$ Institute of Vegetable and Floriculture Science, NARO, Tsu 514-2392, Japan

${ }^{2}$ Graduate School of Science and Technology, Niigata University, Niigata 950-2181, Japan

${ }^{3}$ Graduate School of Agricultural Science, Kobe University, Kobe 657-8501, Japan

Vernalization, a floral transition stimulated by the prolonged exposure to cold temperatures, is an undesirable feature in the breeding of leafy vegetables that impairs productivity by promoting bolting. Cabbage is categorized as a green plant vernalization type; only older plants possess vernalization competence. Despite being of agricultural importance in the breeding of bolting resistance, the molecular mechanism of agedependent acquisition of vernalization competence is poorly understood. In Arabidopsis, FLOWERING LOCUS C (FLC) is a key vernalization response gene that functions as a floral repressor. Its expression decreases upon cold exposure, leading to floral induction. In this study, we characterized three $F L C$ orthologs in cabbage (BoFLC1, BoFLC2, and BoFLC3). Predicted amino acid sequences of the three BoFLC cDNAs were highly conserved with the MADS-box domain. The overexpression of the three BoFLCs in Arabidopsis displayed late flowering phenotypes, indicating the functional conservation of these paralogs as floral repressors in cabbage. The expression analyses before and after constant cold treatment given at different plant ages showed that the three BoFLCs displayed distinct responses to prolonged cold exposure. Regardless of plant age, BoFLC1 declined less markedly, whereas BoFLC2 and BoFLC3 were repressed by sufficient long cold treatment. In both younger and older plants, the expression of the ortholog of floral integrator gene, FLOWERING LOCUS T (FT), was induced by cold treatment, probably due to a reduction in the repressive effect of BoFLCs, suggesting that the fate of age-dependent vernalization is determined independently or downstream of BoFT induction. Our results provide new insights into the mechanism of green plant vernalization in Brassica vegetables.

Key Words: Brassica oleracea, FLC, flowering, green plant vernalization, plant age.

\section{Introduction}

During the plant life cycle, flowering is one of the most crucial events that significantly affects seed productivity. The timing of floral transition is strictly controlled so that fertilization and seed maturation occur in the most suitable conditions. In Arabidopsis, multiple factors have been identified that influence floral transition, including seasonal environmental cues (such as photoperiod and temperature) and endogenous cues

Received; November 9, 2018. Accepted; May 17, 2019.

First Published Online in J-STAGE on July 24, 2019.

This work was supported by a Grant-in-Aid for Scientific Research (B) (15H04433) (JSPS) to K. Okazaki.

* Corresponding author (E-mail: itabashie506@affrc.go.jp). (such as gibberellins [GA], aging, and autonomous factors) (Andrés and Coupland, 2012; Srikanth and Schmid, 2011). Vernalization, an example of floral transition that is promoted by prolonged exposure to cold temperatures, is widely observed in long-day plants of the Brassicacae family. In Arabidopsis, FLOWERING LOCUS C (FLC, AT5G10140) encodes a MADS-box transcription factor and plays a central role in this response (Michaels and Amasino, 1999; Sheldon et al., 1999, 2000). This gene product delays flowering time by repressing the expression of floral integrator genes FLOWERING LOCUS T (FT, AT1G65480) and SUPPRESSOR OF OVEREXPRESSION OF CONSTANS 1 (SOC1, AT2G45660) (Helliwell et al., 2006; Searle et al., 2006). Genetic studies also identified FRIGIDA (FRI, AT4G00650), a major contributor that confers a 
vernalization-requiring habit in a winter-annual accession by activating FLC expression (Johanson et al., 2000; Shindo et al., 2005). When the plant experiences prolonged cold temperatures, the expression of FLC gradually declines, and if the quantitative cold exposure is enough, the repressed state is maintained by epigenetic modifications of histone proteins, even after the surrounding temperature becomes warmer (Bastow et al., 2004; Gendall et al., 2001; Sung et al., 2006). This leads to the de-repression of FT and SOCl and accelerates floral transition and inflorescence development under the subsequently ideal flowering conditions (Helliwell et al., 2006; Searle et al., 2006).

Brassicaceae is one of the most important plant families in horticulture; many economically valuable vegetables belong to it, from which we harvest various parts depending on the variety and species: broccoli (Brassica oleracea L. var. italica) for clusters of florets, rapeseed (Brassica napus L.) for oil-rich seeds, and turnips (Brassica rapa L. var. rapa) for roots. For cabbage (B. oleracea var. capitata), from which the leafy heads are harvested, productivity is impaired in spring due to exposure to cold temperatures in the field throughout the winter months, causing vernalization and promoting bolting before head formation. Thus, breeding of varieties with lower sensitivity to cold temperatures and a high resistance to bolt is desired. To develop reliable DNA markers for marker-assisted breeding, it is important to understand the molecular mechanisms underlying the vernalization response and subsequent floral transition in cabbage.

Vernalization responses in Brassica vegetables are divided into a "seed vernalization type" and a "green plant vernalization type", based on the stage during which the plants are vernalized. Although the seed vernalization type can respond to cold exposure as early as at the imbibed seed stage, the green plant vernalization type needs several weeks for vegetative growth to become competent to be vernalized, and cabbage is categorized as the latter type (Lin et al., 2005; Kuginuki, 2001). The difference in timing of the response to cold temperature is important for breeding. A delay in this timing allows plants to avoid vernalization, which leads to the off-season production of leafy vegetables in spring. In contrast, vernalization at an early stage of vegetative growth can shorten the generation time and accelerate breeding. Despite the importance of this, little is known about the molecular mechanisms involved in this age-dependent vernalization response in Brassica vegetables.

Thus far, to identify the key genes in vernalization, many quantitative trait locus (QTL) studies on flowering properties have been performed to detect loci correlated with agriculturally important traits such as possessing or lacking budding, bolting, and heading in various Brassica species. These analyses revealed the co-localization of QTLs and orthologs for well-known
Arabidopsis flowering genes, such as FLC and FT (Kakizaki et al., 2011; Kitamoto et al., 2014; Kole et al., 2001; Li et al., 2009; Lou et al., 2007; Osborn et al., 1997; Schranz et al., 2002; Xiao et al., 2013; Zhang et al., 2016; Zhao et al., 2010), which suggested the evolutionary conservation of the vernalization pathway mediated by $F L C$ in Brassica species. In the genome of B. oleracea, three copies of FLC orthologous genes (BoFLC1/2/3) and one pseudogene (BoFLC5) have been identified (Okazaki et al., 2007; Schranz et al., 2002). Of these, BoFLC2 located on chromosome 2 , has been found to function as a floral repressor and to contribute to the vernalization response (Irwin et al., 2016; Lin et al., 2005; Okazaki et al., 2007; Ridge et al., 2015; Shea et al., 2018). However, the functions of two other BoFLC genes (BoFLC1/3) remain largely unknown.

To understand the molecular basis of age-dependent vernalization, it is first necessary to determine which step of vernalization is age-dependent. In this study, we first examined $B o F L C 1 / 3$ and $B o F L C 2$ overexpression in Arabidopsis to determine if they could function as floral repressors. Furthermore, to consider the relationship between cold response and plant age, we performed expression analyses of vernalization-related genes during cold treatment and compared their expression dynamics before and after acquisition of vernalization competence. Our results provide new insights into green plant vernalization in cabbage and provide beneficial information on the breeding of bolting resistance in Brassica vegetables.

\section{Materials and Methods}

\section{Plant materials and growth conditions}

Using a genetically fixed plant as a material enables cloning of uniform nucleotide sequences with certainty. Thus, we chose to use MP-22, a doubled haploid (DH) line derived from a commercial $F_{1}$ hybrid cultivar of the cabbage 'Matsunami' (Ishii Seed Growers, Japan). MP-22 was obtained by chromosome doubling that naturally occurred upon tissue culture of haploid plants derived from isolated microspores (Kuginuki et al., 1999). The doubled chromosomes were observed under a microscope and seed fertility after self-pollination was confirmed (unpublished data).

All plants were grown in plastic pots $150 \mathrm{~mm}$ in diameter in an air-conditioned room. Plants without cold treatment were grown under ambient temperature and long-day (LD) conditions ( $16 \mathrm{~h} \mathrm{light}$ at $25^{\circ} \mathrm{C} / 8 \mathrm{~h}$ dark at $18^{\circ} \mathrm{C}$ ). Plants treated with prolonged cold temperature were prepared as follows; (1) ambient temperature under LD condition $\left(16 \mathrm{~h}\right.$ light at $25^{\circ} \mathrm{C} / 8 \mathrm{~h}$ dark at $\left.18^{\circ} \mathrm{C}\right)$, (2) followed by prolonged cold treatment $(12 \mathrm{~h}$ light at $5^{\circ} \mathrm{C} / 12 \mathrm{~h}$ dark at $5^{\circ} \mathrm{C}$ ) for three, five, and, eight weeks, and (3) and returning to ambient temperature $\left(16 \mathrm{~h}\right.$ light at $25^{\circ} \mathrm{C} / 8 \mathrm{~h}$ dark at $\left.18^{\circ} \mathrm{C}\right)$ for a further growth period of two weeks. The age at which plants 
were transferred to cold conditions was determined based on the leaf number. Arabidopsis plants were grown in a growth chamber set at $22^{\circ} \mathrm{C}$ with a $16 \mathrm{~h}$ light $/ 8 \mathrm{~h}$ dark cycle.

\section{Cloning of BoFLC and sequencing}

Total RNA was isolated from the youngest leaf of MP-22 at the 14-leaf stage without cold treatment. cDNA was synthesized using a PrimeScript 1st strand cDNA Synthesis Kit (Takara Bio, Japan) in accordance with the manufacturer's instructions. DNA digestion of $500 \mathrm{ng}$ of total RNA was performed with the enzyme solution included in the kit. RT-PCR was performed using PrimeSTAR GXL DNA Polymerase (Takara Bio) under the following conditions: 1 cycle at $98^{\circ} \mathrm{C}$ for $5 \mathrm{~s}$, 30 cycles of $98^{\circ} \mathrm{C}$ for $10 \mathrm{~s}, 55^{\circ} \mathrm{C}$ for $15 \mathrm{~s}$, and $68^{\circ} \mathrm{C}$ for $1 \mathrm{~min}$, and a final extension at $68^{\circ} \mathrm{C}$ for $2 \mathrm{~min}$. After Atailing, RT-PCR products cloned into pGEM-T Easy vector (Promega, USA) were transformed into Escherichia coli TOP10 competent cells. The plasmid isolated by QIAprep Spin Miniprep Kit (QIAGEN, Germany) was sequenced using a BigDye Terminator v3.1 Cycle Sequencing Kit (Thermo Fisher Scientific, USA). Primers used in the above experiments are listed in Table S1.

\section{Overexpression of BoFLCs and BoFT}

Total RNA was isolated from the youngest leaves of the plants, and cDNA was synthesized using a PrimeScript 1st strand cDNA Synthesis Kit (Takara Bio). The construction was carried out by inserting the cDNA fragment covering 5'UTR to 3 'UTR into the downstream $35 \mathrm{~S}$ promoter in a pCAMBIA1301 binary vector. cDNAs of BoFLCs and BoFT were derived from plants before and after eight weeks of cold treatment at the 14-leaf stage, respectively. The Agrobacterium tumefaciens Gv3101 strain with an overexpression construct was used to infect Arabidopsis Col-0 and C24 using the floral-dip method. Selection of transformants was achieved on 1/2 Murashige and Skoog media (Nihon Pharmaceutical, Japan) including 1\% sucrose and $20 \mathrm{mg} \cdot \mathrm{L}^{-1}$ of hygromycin B (Wako, Japan). The insertion of transgenes was confirmed by PCR using primer pairs specific to each construct and the hygromycin phosphotransferase ( $h p t$ ) gene. The flowering time of Arabidopsis was assessed based on the number of leaves at the bolting stage.

\section{Expression analyses of flowering genes}

Total RNA was isolated using an RNeasy Plant Mini Kit (QIAGEN) from the youngest leaves of the plants grown as follows (Fig. 1): (1) NV, ambient temperature before cold treatment $\left(16 \mathrm{~h}\right.$ light at $25^{\circ} \mathrm{C} / 8 \mathrm{~h}$ dark at $\left.18^{\circ} \mathrm{C}\right)$, (2) $3 \mathrm{~V} / 8 \mathrm{~V}$, cold treatment $\left(12 \mathrm{~h}\right.$ light at $5^{\circ} \mathrm{C} / 12 \mathrm{~h}$ dark at $5^{\circ} \mathrm{C}$ ) for three or eight weeks, and (3) $8 \mathrm{VN}$, ambient temperature for a further two weeks after eight weeks of cold treatment. cDNA synthesis was carried
A

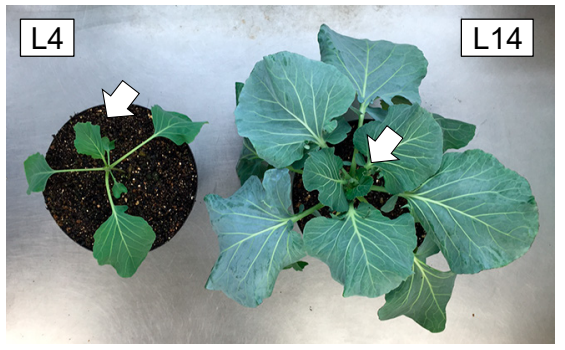

B
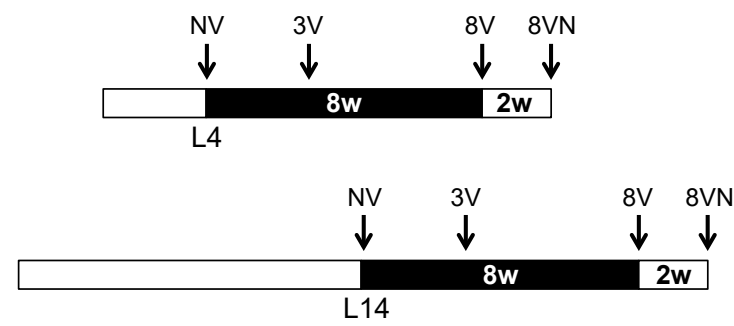

Fig. 1. Cabbage plants grown in an air-conditioned room. A: MP-22 at different ages. Leaf number was used as the criterion to define plant age. L4 and L14 represent the 4- and 14-leaf stages. Arrows indicate leaves collected for RNA extraction. B: Time points of sample collection for expression analyses are indicated by arrows at the 4-leaf stage (L4, upper diagram), and the 14-leaf stage (L14, lower diagram). White box and black box represent the ambient and cold temperature conditions, respectively. NV, without cold treatment; $3 \mathrm{~V}$, with three weeks of cold treatment; $8 \mathrm{~V}$, with eight weeks of cold treatment; $8 \mathrm{VN}$, with eight weeks of cold treatment followed by two weeks' growth under the ambient temperature.

out using a PrimeScript 1st strand cDNA Synthesis Kit (Takara Bio). DNA digestion of $500 \mathrm{ng}$ of total RNA was performed with the enzyme solution included in the kit. RT-qPCR was performed with Thermal Cycler Dice Real Time System II (Takara Bio) using SYBR Premix Ex Taq II (Takara Bio). BoACTIN (Bol024627) at the Bolbase (http://119.97.203.210/bolbase/index.html) was used as an internal control. This experiment was performed with three biological replicates.

To estimate the relative expression of BoFLC genes, RT-PCR was performed using Quick Taq HS DyeMix (TOYOBO, Japan) under the following conditions: 1 cycle at $94^{\circ} \mathrm{C}$ for $2 \mathrm{~min}, 29$ cycles of $94^{\circ} \mathrm{C}$ for $30 \mathrm{~s}$, $55^{\circ} \mathrm{C}$ for $30 \mathrm{~s}$, and $68^{\circ} \mathrm{C}$ for $30 \mathrm{~s}$, and a final extension at $68^{\circ} \mathrm{C}$ for $2 \mathrm{~min}$. RT-PCR products cloned into pGEM-T Easy vector (Promega) were transformed into E. coli TOP10 competent cells. Among 60 colonies, those containing each BoFLC gene were counted by direct sequencing using a BigDye Terminator v3.1 Cycle Sequencing Kit (Thermo Fisher Scientific). A primer set that commonly amplifies four BoFLC genes was used for RT-PCR, and an M13 forward primer was used for the sequencing. This experiment was performed with two biological replicates. Primer sequences used for the expression analysis are shown in Table S1. 


\section{Results}

Characterization of key vernalization response genes in B. oleracea

In Arabidopsis, a floral repressor gene, FLOWERING $L O C U S C(F L C)$ plays a central role in the vernalization response. To clarify whether cabbage FLC orthologs produce comparable effects to $F L C$ on flowering time, we first sequenced the coding regions of BoFLCs in our plant material. In MP-22, cDNAs of three copies of FLC orthologs (BoFLC1/2/3) could be amplified by RT-PCR using locus-specific primers, and BoFLC5 cDNA was not amplified, which was consistent with previous reports in other $B$. oleracea cultivars indicating that BoFLC5 may be a pseudogene (data not shown, Okazaki et al., 2007). There are several reports in which different synonyms were used to represent BoFLC in chromosome 2, such as BoFLC4, BoFLC2, and BoFLC.C2 (Irwin et al., 2016; Lin et al., 2005; Okazaki et al., 2007; Ridge et al., 2015; Shea et al., 2018). In this study, we named it BoFLC2, following Okazaki et al. (2007). Sequencing of BoFLC cDNAs, except $B o F L C 5$, in MP-22 revealed that BoFLC1, BoFLC2, and BoFLC3 ORFs were all $594 \mathrm{bp}$ in length and encoded 197 amino acids. There were no apparent polymorphisms that may cause loss-of-function mutations in $B o F L C 1, B o F L C 2$, or BoFLC3, such as the single base deletion in exon 4 of $B o F L C 2$ thought to be responsible for early flowering in a non-vernalization-type cultivar of broccoli (Okazaki et al., 2007). The similarities of their predicted amino acid sequences to FLC were $84.42 \%, 80.90 \%$, and $82.91 \%$, respectively, and the MADS domains - important for DNA-binding abilitywere well conserved (Figs. 2 and S1). To confirm the functional conservation as floral repressors of $B o F L C$ gene products, we made transgenic Arabidopsis plants carrying the construct to overexpress BoFLC1, $B o F L C 2$, or BoFLC3 cDNA driven by the CaMV $35 \mathrm{~S}$ promoter. We eliminated BoFLC5 as it was deemed to be a pseudogene from our overexpression analysis. We

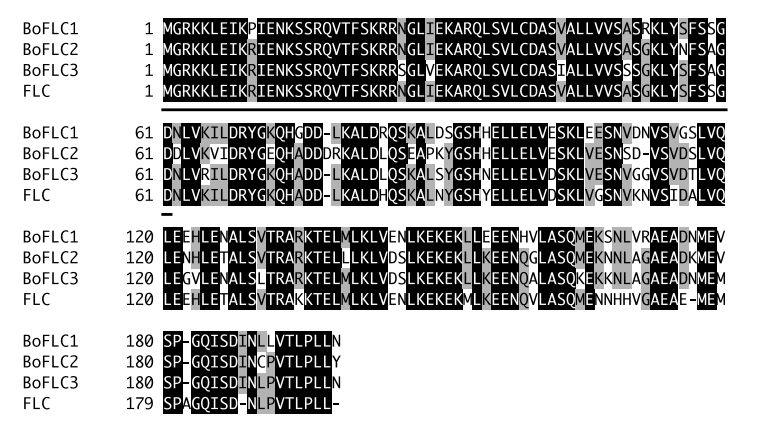

Fig. 2. Alignment of predicted amino acid sequences of FLC and BoFLCs. CLUSTALW was used as the alignment program. The MADS-box domain predicted by the Conserved Domain Database (CDD) of the National Center for Biotechnology Information (NCBI; https://www.ncbi.nlm.nih.gov/cdd) is underlined. chose Col-0 as a background, a rapid-flowering accession with low FLC expression owing to its lack of FRI activity (Shindo et al., 2005). We obtained 37, 38, and 47 hygromycin-resistant $\mathrm{T}_{1}$ plants possessing BoFLC1, $B o F L C 2$, and BoFLC3 cDNA, respectively, and insertion of the transgenes was confirmed by PCR using specific primer pairs. Next, we examined the flowering time measured by the leaf number at the bolting stage. Compared with the population of 25 wild-type plants, the leaf number distribution in transgenic lines for three constructs was shifted to a larger value (Fig. 3A). Furthermore, some extremely late flowering lines appeared in the $\mathrm{T}_{1}$ generation, bolting at least 180 days after sowing (Fig. 3B-F), and their late flowering features were inherited by the next generation (Fig. S2). These observations indicated that $B o F L C$ paralogs of MP-22 could delay the budding and flowering time in cabbage as FLC does in Arabidopsis (Michaels and Amasino, 1999; Sheldon et al., 1999, 2000).

\section{Age-dependent vernalization response in cabbage}

To estimate the age at which MP-22 vernalizes in response to constant cold temperature, we measured the ratio of plants that formed flower buds after five and eight weeks of cold treatment. MP-22 plants were exposed to either five or eight weeks of cold treatment that was started at the 4-, 10-, 14-, or 20-leaf stages of vegetative growth. At the end of cold treatment, the plants were then returned to growth conditions and monitored for 60 days. Table S2 shows the ratio of plants that showed flower bud formation for each treatment. When the cold treatment was started at the 4- and 10-leaf stages, none of the six plants tested formed flower buds, even 60 days after returning to the ambient temperature. In contrast, when the cold treatment was started at the 14-leaf stage, flower bud formation was observed for all six plants within 30 days after the end of the cold treatment. These results indicated that MP-22 needs to reach at least the 14-leaf stage to fully respond to vernalization treatment. We also confirmed that eight weeks of cold treatment was long enough to stimulate flower bud formation, as plants at the 14- and 20-leaf stage were fully vernalized by five weeks of cold treatment (Table S2). Therefore, we suggest using less than 10-leaf, and more than 14-leaf, stage plants as juvenile and adult-age plants, respectively, based on being with or without competence to be vernalized under our growth conditions. In this study, all experiments were performed for 4-leaf-stage and 14-leafstage plants, as juvenile and adult plants, respectively.

\section{Expression analysis of three BoFLCs before/after ver- nalization}

We decided to examine whether floral transition was correlated to the expression of BoFLC genes after prolonged cold exposure in MP-22. We assessed whether the expression level of BoFLCs decreased after three 
and eight weeks of prolonged cold treatment as shown in FLC. We monitored the expression profiles of three genes individually and found that they responded differently to cold temperature (Fig. 4A-C). In the adult plants, the transcript of BoFLC2 decreased gradually with the length of cold exposure (Fig. 4B), while the expression of BoFLC3 exhibited a more dramatic reduction with the cold treatment (Fig. 4C). Whereas the above two genes exhibited considerable downregulation with cold temperatures, the expression of BoFLC1
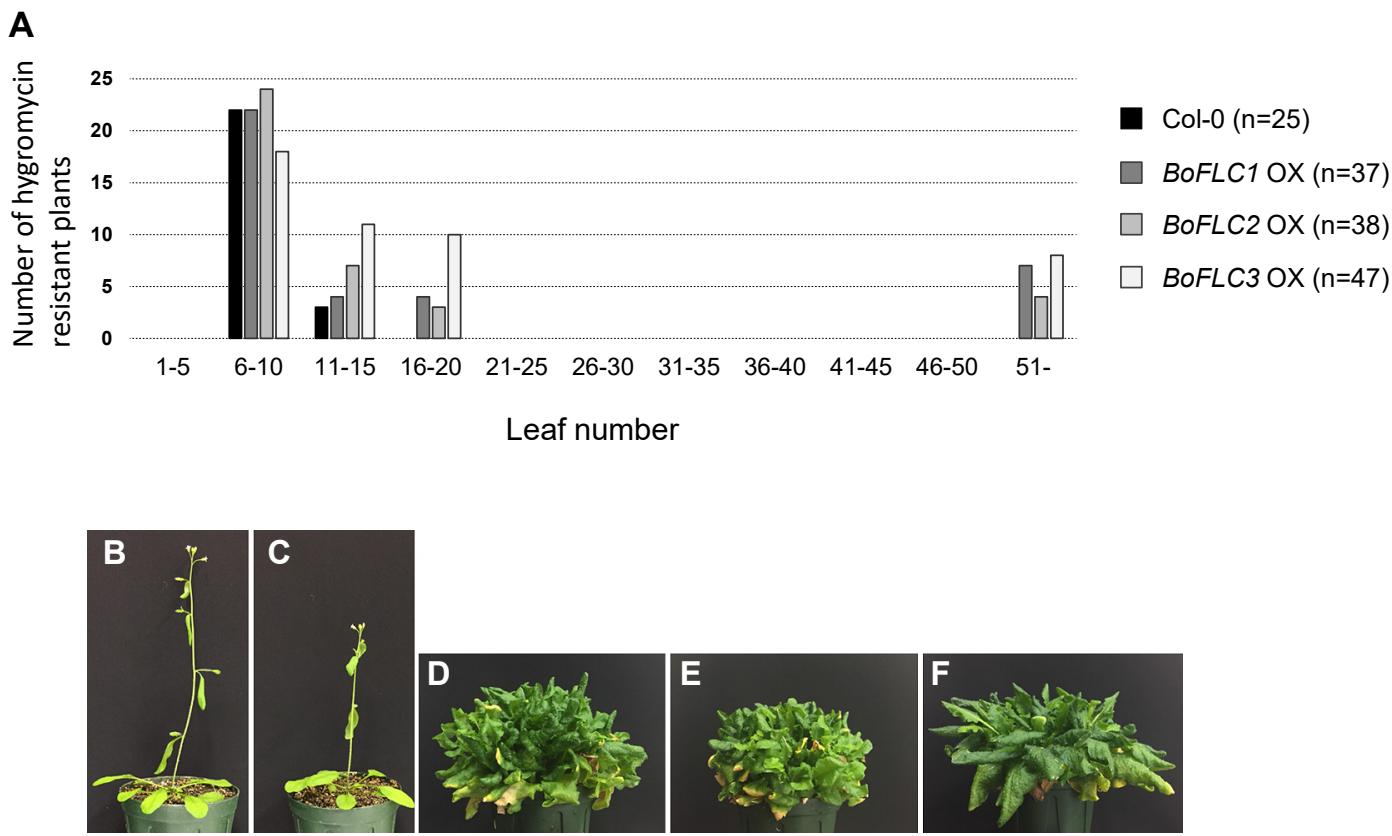

Fig. 3. Late flowering phenotype in Arabidopsis overexpressing BoFLC genes with the Col-0 background. A: The distribution of leaf number counted at the bolting stage. B-F: Transgenic Arabidopsis showing extremely late flowering that bolted at more than the 51-leaf stage. (B) Wild-type plant of Col-0 and (C) a transgenic plant possessing only the pCAMBIA1301 vector are shown as controls. Some overexpression lines of (D) BoFLC1, (E) BoFLC2, and (F) BoFLC3 in the $\mathrm{T}_{1}$ generation did not bolt even at 180 days after sowing.

A

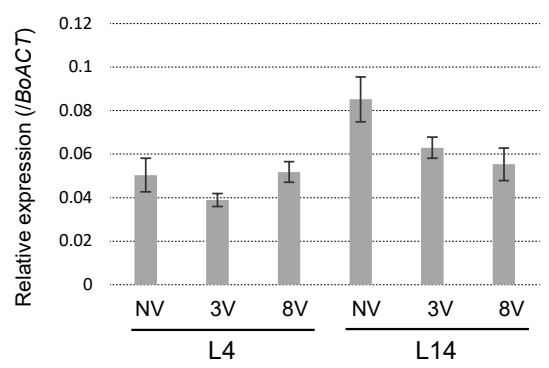

C

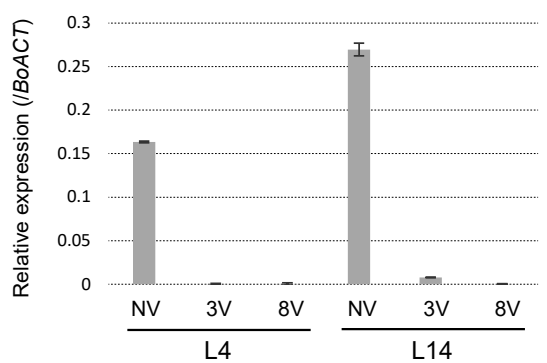

B



D

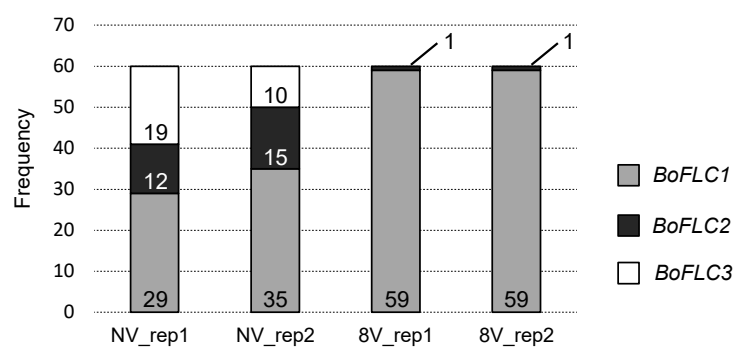

Fig. 4. Gene expression profiles against prolonged cold treatment started at different ages. A, B, and C: Expression levels of BoFLC1, BoFLC2, and BoFLC3 at the end of different periods of vernalization. D: Relative expression level among BoFLC paralogues at the 4-leaf stage. Numbers inside bars represent the frequency of each clone. L4, the 4-leaf stage; L14, the 14-leaf stage. NV, without cold treatment; 3V, with three weeks of cold treatment; $8 \mathrm{~V}$, with eight weeks of cold treatment. 
decreased only moderately (Fig. 4A). In the juvenile plants, both $B o F L C 2$ and $B o F L C 3$ were repressed after eight weeks of cold treatment. However, in contrast to the transcript of BoFLC3 decreasing rapidly, the same as for the adult plants, the expression of BoFLC2 was less markedly affected (Figs. 4B, C, and S4B). At the juvenile age, the expression of BoFLCl slightly decreased after three weeks of cold treatment, but during a further five weeks of cold treatment it increased again up to a level comparable with that before cold treatment.

When we focused on the reduction rate of transcripts after the cold treatment, the expression profiles of BoFLC1 and BoFLC2 were age-dependent (Fig. S4). In the adult plants, the transcript level of BoFLC2 declined sharply compared with the juvenile plants, and the difference was observed relatively early in the process of cold treatment (Fig. S4B). However, a difference in the expression profile of $B o F L C 1$ between the juvenile and adult plants was seen relatively later in the process of cold response; namely, different from the adult plants, the repression of BoFLCl after three weeks of cold treatment was not maintained in the juvenile plants (Fig. S4A).

Next, to more directly compare the expression profiles and quantitative balance of transcripts for BoFLC paralogs, the relative expression levels of individuals were estimated based on their abundance ratios in RTPCR products. RT-PCR was performed using a primer pair common to four orthologs (BoFLC1/2/3/5) (Table S1) and the PCR program was set with 29 cycles so as not to reach a plateau. After vector cloning and transformation into $E$. coli, we counted the number of colonies, including plasmids, with each BoFLC sequence.

In the juvenile plants, RT-PCR products were visible on agarose gel to be cloned even after eight weeks of cold treatment, suggesting that the total transcript dosage of BoFLC paralogs was less sensitive to vernalization. Before cold treatment, it was estimated that the expression of BoFLCl was the highest among the four paralogues (Fig. 4D), and after eight weeks of cold treatment, more than $98 \%$ of RT-PCR products were occupied by the BoFLC1 clone, suggesting that this gene was less sensitive to cold treatment (Fig. 4D). It is probable that the lower sensitivity to cold temperatures of the total transcript dosage of $B o F L C$ s is due to BoFLC1. No colonies containing the BoFLC5 clone were detected in any conditions, consistent with the assumption that BoFLC5 may be a pseudogene.

Expression analysis of flowering genes that may function after BoFLCs repression

The difference in expression profiles of BoFLC1 and BoFLC2 between the juvenile and adult plants (Figs. 4 and S4) motivated us to consider the possibility that there were factors regulating the expression of $B o F L C 1$ and $B o F L C 2$ under cold temperatures, and that their activities were age-dependent. Next, we decided to examine the expression of orthologs of VERNALIZATION INSENSITIVE 3 (VIN3, AT5G57380), one of the key players in epigenetic silencing of FLC in Arabidopsis, and its expression is transiently increased during prolonged cold exposure (Sung and Amasino, 2004). At least two copies of BoVIN3 (Bol026036 and Bol014227) were encoded in the B. oleracea genome. RT-qPCR using a primer pair to commonly amplify two BoVIN3 genes showed that the expression was induced only during exposure to cold temperature (Fig. 5A), implying that epigenetic regulation via histone modification occurred during cold treatment in cabbage. However, this upregulation was observed regardless of plant age, suggesting that with or without cold-induced expression, BoVIN3 was not related to the agedependency of vernalization in cabbage.

We further examined the expression of genes predicted to function downstream of $B o F L C$ repression. In Arabidopsis, FT is one of the target genes of FLC, and it is repressed through direct binding of FLC to regulatory elements (Helliwell et al., 2006). Two FT orthologous genes are encoded in the genome of B. oleracea on chromosome 2 (Bol039209) and chromosome 6 (Bol012573), and it has been reported that BoFT on chromosome 2 was not transcribed (Wang et al., 2012).

A

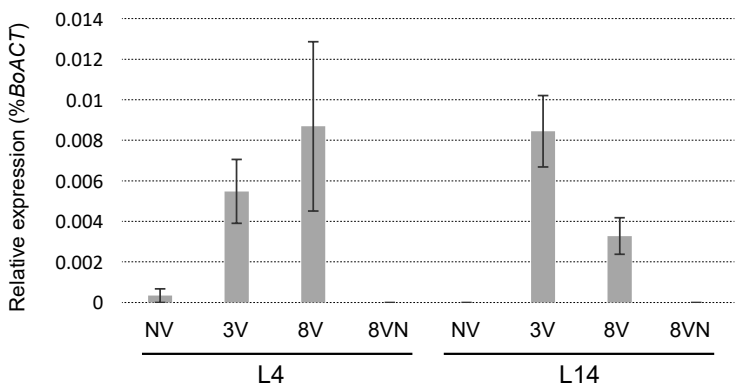

B

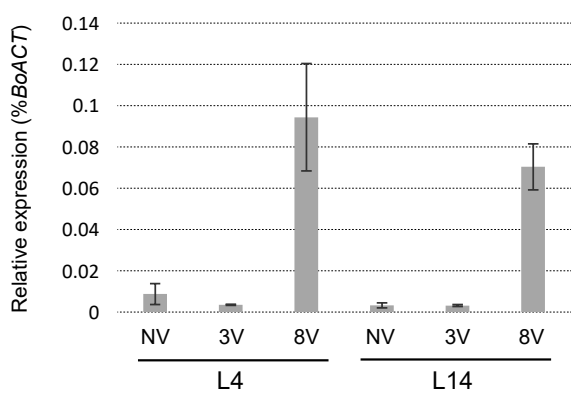

Fig. 5. Gene expression profiles against prolonged cold treatment at different ages. Quantitative RT-PCR was performed for (A) BoVIN3 and (B) BoFT. L4, the 4-leaf stage; L14, the 14-leaf stage. NV, without cold treatment; $3 \mathrm{~V}$, with three weeks of cold treatment; $8 \mathrm{~V}$, with eight weeks of cold treatment; $8 \mathrm{VN}$, with eight weeks cold treatment followed by two more weeks of growth under the ambient temperature. 
We decided to focus on BoFT on chromosome 6 and to overexpress this gene in the $\mathrm{C} 24$ (FRI/FLC) background. This genetic background is suitable because endogenous $F T$ is repressed by the action of FLC. Overexpression of BoFT showed an early flowering phenotype compared with WT plants, suggesting the function of the FT ortholog in MP-22 as a floral integrator gene was conserved (Fig. S3). We performed RTqPCR for plants with or without cold treatment started at different plant ages. In the adult plants, it was shown that the expression of BoFT was induced in plants by eight weeks of cold treatment, but not for plants exposed to less than three weeks of treatment (Fig. 5B), suggesting that the repressed state of BoFT was slightly released owing to a reduction in the repressive effect of BoFLCs due to the eight weeks of cold exposure. Interestingly, a similar profile was also observed in the juvenile plants without the competence to be vernalized. These results suggested that the age-dependent vernalization response may be controlled via pathways independent or downstream of BoFT upregulation. The above results also suggested that upregulation of $B o F T$ can occur even when BoFLC1 expression remains. It is possible that repressive activity, or binding affinity, to target sequences is weaker than that of $B o F L C 2$ and BoFLC3.

\section{Discussion}

Prolonged exposure to cold temperature is one of the cues that affect flowering time. Understanding the molecular mechanisms of the vernalization response in cabbage, and the differences between cultivars, is important for the development of DNA markers for the breeding of varieties insensitive to cold temperature. In this study, to provide new insight into the molecular basis of green plant vernalization, we tried to determine which process was age-dependent during the vernalization response and correlated to the fate of subsequent flower bud formation.

Using a genetically fixed $\mathrm{DH}$ line derived from a commercial $\mathrm{F}_{1}$ cultivar named MP-22, we first sequenced three BoFLC cDNAs (BoFLC1/2/3) whose predicted products harbor the MADS-box domain. Arabidopsis plants overexpressing BoFLCs with the Col-0 background, in which the endogenous FLC expression is quite low, showed a late flowering phenotype. Thus far, among BoFLC orthologs, BoFLC2 has been mainly recognized for its contribution to vernalization. However, we suggest that every BoFLC gene should be considered because the functional conservation of three $B o F L C$ genes as floral repressors was shown in this study.

For three constructs, the frequency of extremely late flowering plants among hygromycin-resistant plants was low. This result is similar to a previous report of overexpression of FLC in Col-0; a small number of transformants displayed markedly late flowering, but the majority flowered early or comparably to the WT (Ratcliffe et al., 2001). In this case, the late-flowering plants highly expressed the transgene compared with early flowering plants. Furthermore, there was also a report that Arabidopsis (FRI/FLC) with extra copies of the $F L C$ transgene did not flower without cold treatment, similar to true biennials (Michaels and Amasino, 2000). It is possible that BoFLCs act in a dosagedependent manner similar to $F L C$, and that the markedly late flowering in our experiment was due to a greater amount of BoFLC protein, which may interact with floral activators as well as $F T$ and $S O C 1$ and lead to unusual titration.

Age-dependent vernalization was confirmed for MP-22 under our growth conditions and plants needed to reach at least the 14-leaf stage to be vernalized in eight weeks of prolonged cold treatment. We also examined the expression of three BoFLC genes before and after cold treatment at different plant ages and found that they responded differently to cold temperature. In contrast to BoFLC2 and BoFLC3, both of which were downregulated by cold treatment in the juvenile and adult plants, BoFLC1 was less sensitive to cold temperature. When we focused on the reduction rate of transcripts during cold response, the expression profiles of BoFLC1 and BoFLC2 seemed to be age-dependent (Figs. 4 and S4). This indicated the presence of transacing factors that affected the expression of these two genes and that their effects may be age-dependent. The difference in regulation of cold response between the two ages was likely to be observed in the earlier and later process of vernalization for $B o F L C 2$ and $B o F L C 1$, respectively (Fig. S4). There may be age-dependent mechanisms that distinctly regulate the expression of either BoFLC1 or BoFLC2, and their effects can be seen at different stages during the cold response.

Further expression analysis of BoFT, which is predicted to be a target of BoFLCs, showed that it was upregulated by eight weeks of cold treatment regardless of plant age. This may be a result of decreased repressive effects of BoFLCs and suggests that flower bud formation was dependent on processes downstream of BoFT upregulation or was determined independently by the vernalization-mediated flowering pathway. Our expression analysis suggested that de-repression of BoFT can occur even if the expression of $B o F L C 1$ remains after eight weeks of cold treatment. However, Arabidopsis plants overexpressing BoFLCl were late flowering. One possible explanation for this discrepancy is that the repression of BoFT by the action of BoFLCl, but not $B o F L C 2 / 3$, could be specifically disrupted by derivatives of other loci whose equivalents are not present in Arabidopsis (Col-0). Even if the transcript level per cell was comparable between the juvenile and adult plants, the juvenile plants harboring fewer leaves may eventually supply less BoFT protein to the shoot apex. If it is under the threshold required for downstream processes 
to trigger floral transition, age-dependent flower bud formation could be explained by failure of BoFT to sufficiently interact with partners to regulate direct target gene(s).

The molecular mechanisms of age-dependent vernalization have also been investigated in perennial Arabis alpina L., a close relative of Arabidopsis. In this species, an FLC ortholog, PERPETUAL FLOWERING1 (PEP1, KC123238.1), and an SOC1 ortholog are downand up-regulated, respectively, by prolonged cold exposure regardless of plant age (Wang et al., 2011). It was also suggested that an age-dependent flowering pathway, other than the vernalization pathway, acted as a determinant of competency to induce floral transition during cold exposure (Bergonzi et al., 2013; Wu et al., 2009; Xu et al., 2016). Our result was similar to the observation in this perennial in terms of the upregulation of genes targeted by the FLC ortholog and was independent of plant age. It is therefore important to consider the possibility that there is an overlap of the mechanism of age-dependent vernalization between the two species.

For efficient breeding of leafy vegetables highly resistant to bolt, it is important to design reliable DNA markers linked to delayed acquisition of vernalization competence. It is known that the timing to respond to cold temperature in the field largely depends on the genetic background of Japanese cabbage cultivars (Kuginuki, 2001). Indeed, under our growth conditions, in plants that were grown in plastic pots in an airconditioned room, a difference in the developmental stage of vernalization between several cultivars was confirmed. Whereas MP-22 became vernalized between the 10- and 14-leaf stages, several cultivars originating from the Copenhagen market cabbage responded to cold treatment earlier than the 7-leaf stage (unpublished data). Furthermore, among Japanese varieties, there are some commercial $\mathrm{F}_{1}$ cultivars that respond to cold temperature at quite late stages, such as 'Kinkei-201' (Sakata Seed, Japan). The identification of allele(s) that correspond to the delay in acquisition of vernalization competence in these cultivars will be of benefit in the design of DNA markers and enable control of vernalization timing based on marker-assisted breeding. Therefore, further analyses are required to identify novel factors and uncover the mode of action involved in the complex flowering mechanisms that may be governed by multiple pathways in Brassica vegetables.

\section{Literature Cited}

Andrés, F. and G. Coupland. 2012. The genetic basis of flowering responses to seasonal cues. Nat. Rev. Gebet. 13: 627-639.

Bastow, R., J. S. Mylne, C. Lister, Z. Lippman, R. A. Martienssen and C. Dean. 2004. Vernalization requires epigenetic silencing of FLC by histone methylation. Nature 427: 164-167.

Bergonzi, S., M. C. Albani, E. Ver Loren van Themaat, K. J. Nordström, R. Wang, K. Schneeberger, P. D. Moerland and G. Coupland. 2013. Mechanisms of age-dependent response to winter temperature in perennial flowering of Arabis alpina. Science 340: 1904-1907.

Gendall, A. R., Y. Y. Levy, A. Wilson and C. Dean. 2001. The VERNALIZATION 2 gene mediates the epigenetic regulation of vernalization in Arabidopsis. Cell 107: 525-535.

Helliwell, C. A., C. C. Wood, M. Robertson, W. J. Peacock and E. S. Dennis. 2006. The Arabidopsis FLC protein interacts directly in vivo with $S O C 1$ and $F T$ chromatin and is part of a high-molecular-weight protein complex. Plant J. 46: 183192.

Irwin, J. A., E. Soumpourou, C. Lister, J. D. Ligthart, S. Kennedy and C. Dean. 2016. Nucleotide polymorphism affecting FLC expression underpins heading date variation in horticultural brassicas. Plant J. 87: 597-605.

Johanson, U., J. West, C. Lister, S. Michaels, R. Amasino and C. Dean. 2000. Molecular analysis of FRIGIDA, a major determinant of natural variation in Arabidopsis flowering time. Science 290: 344-347.

Kakizaki, T., T. Kato, N. Fukino, M. Ishida, K. Hatakeyama and S. Matsumoto. 2011. Identification of quantitative trait loci controlling late bolting in Chinese cabbage (Brassica rapa L.) parental line Nou 6 gou. Breed. Sci. 61: 151-159.

Kitamoto, N., S. Yui, K. Nishikawa, Y. Takahata and S. Yokoi. 2014. A naturally occurring long insertion in the first intron in the Brassica rapa FLC2 gene caused delayed bolting. Euphytica 196: 213-223.

Kole, C., P. Quijada, S. D. Michaels, R. M. Amasino and T. C. Osborn. 2001. Evidence for homology of flowering-time genes VFR2 from Brassica rapa and FLC from Arabidopsis thaliana. Theor. Appl. Genet. 102: 425-430.

Kuginuki, Y. 2001. Cabbage. p. 796-817. In: S. Nishi (ed.). Shinpen yasai engei handbook (In Japanese). Yokendo, Tokyo.

Kuginuki, Y., T. Miyajima, H. Masuda, K. Hida and M. Hirai. 1999. Highly regenerative cultivars in microspore culture in Brassica oleracea L. var. capitata. Breed. Sci. 49: 251-256.

Li, F., H. Kitashiba, K. Inaba and T. Nishio. 2009. A Brassica rapa linkage map of EST-based SNP markers for identification of candidate genes controlling flowering time and leaf morphological traits. DNA Res. 16: 311-323.

Lin, S.-I., J.-G. Wang, S.-Y. Poon, C.-L. Su, S.-S. Wang and T.-J. Chiou. 2005. Differential regulation of FLOWERING LOCUS $C$ expression by vernalization in cabbage and Arabidopsis. Plant Physiol. 137: 1037-1048.

Lou, P., J. Zhao, J. S. Kim, S. Shen, D. P. Del Carpio, X. Song, M. Jin, D. Vreugdenhil, X. Wang, M. Koornneef and G. Bonnema. 2007. Quantitative trait loci for flowering time and morphological traits in multiple populations Brassica rapa. J. Exp. Bot. 58: 4005-4016.

Michaels, S. D. and R. M. Amasino. 1999. FLOWERING LOCUS $C$ encodes a novel MADS domain protein that acts as a repressor of flowering. Plant Cell 11: 949-956.

Michaels, S. D. and R. M. Amasino. 2000. Memories of winter: vernalization and the competence to flower. Plant Cell Environ. 23: 1145-1154.

Okazaki, K., K. Sakamoto, R. Kikuchi, A. Saito, E. Togashi, Y. Kuginuki, S. Matsumoto and M. Hirai. 2007. Mapping and characterization of FLC homologs and QTL analysis of flowering time in Brassica oleracea. Theor. Appl. Genet. 114: 595-608.

Osborn, T. C., C. Kole, I. A. Parkin, A. G. Sharpe, M. Kuiper, D. J. Lydiate and M. Trick. 1997. Comparison of flowering time genes in Brassica rapa, B.napus and Arabidopsis thaliana. Genetics 146: 1123-1129.

Ratcliffe, O. J., G. C. Nadzan, T. L. Reuber and J. L. Riechmann. 
2001. Regulation of flowering in Arabidopsis by an FLC homologue. Plant Physiol. 126: 122-132.

Ridge, S., P. H. Brown, V. Hecht, R. G. Driessen and J. L. Weller. 2015. The role of BoFLC2 in cauliflower (Brassica oleracea var. botrytis L.) reproductive development. J. Exp. Bot. 66: $125-135$.

Schranz, M. E., P. Quijada, S. B. Sung, L. Lukens, R. Amasino and T. C. Osborn. 2002. Characterization and effects of the replicated flowering time gene FLC in Brassica rapa. Genetics 162: 1457-1468.

Searle, I., Y. He, F. Turck, C. Vincent, F. Fornara, S. Kröber, R. M. Amasino and G. Coupland. 2006. The transcription factor FLC confers a flowering response to vernalization by repressing meristem competence and systemic signaling in Arabidopsis. Genes Dev. 20: 898-912.

Shea, D. J., Y. Tomaru, E. Itabashi, Y. Nakamura, T. Miyazaki, T. Kakizaki, T. N. Naher, M. Shimizu, R. Fujimoto, E. Fukai and K. Okazaki. 2018. The production and characterization of a BoFLC2 introgressed Brassica rapa by repeated backcrossing to an $F_{1}$. Breed. Sci. 68: 316-325.

Sheldon, C. C., J. E. Burn, P. P. Perez, J. Metzger, J. A. Edwards, W. J. Peacock and E. S. Dennis. 1999. The FLF MADS box gene: a repressor of flowering in Arabidopsis regulated by vernalization and methylation. Plant Cell 11: 445-458.

Sheldon, C. C., D. T. Rouse, E. J. Finnegan, W. J. Peacock and E. S. Dennis. 2000. The molecular basis of vernalization: the central role of FLOWERING LOCUS C (FLC). Proc. Natl. Acad. Sci. USA 97: 3753-3758.

Shindo, C., M. J. Aranzana, C. Lister, C. Baxter, C. Nicholls, M. Nordborg and C. Dean. 2005. Role of FRIGIDA and FLOWERING LOCUS $C$ in determining variation in flowering time of Arabidopsis. Plant Physiol. 138: 11631173.

Srikanth, A. and M. Schmid. 2011. Regulation of flowering time: all roads lead to Rome. Cell. Mol. Life Sci. 68: 2013-2037.

Sung, S. and R. M. Amasino. 2004. Vernalization in Arabidopsis thaliana is mediated by the PHD finger protein VIN3.
Nature 427: 159-164.

Sung, S., Y. He, T. W. Eshoo, Y. Tamada, L. Johnson, K. Nakahigashi, K. Goto, S. E. Jacobsen and R. M. Amasino. 2006. Epigenetic maintenance of the vernalized state in Arabidopsis thaliana requires LIKE HETEROCHROMATIN PROTEIN 1. Nat. Genet. 38: 706-710.

Wang, J., C. J. Hopkins, J. Hou, X. Zou, C. Wang, Y. Long, S. Kurup, G. J. King and J. Meng. 2012. Promoter variation and transcript divergence in Brassicaceae lineages of FLOWERING LOCUS T. PLoS ONE 7: e47127.

Wang, R., M. C. Albani, C. Vincent, S. Bergonzi, M. Luan, Y. Bai, C. Kiefer, R. Castillo and G. Coupland. 2011. AaTFL1 confers an age-dependent response to vernalization in perennial Arabis alpina. Plant Cell 23: 1307-1321.

Wu, G., M. Y. Park, S. R. Conway, J.-W. Wang, D. Weigel and R. S. Poething. 2009. The sequential action of miR156 and miR172 regulates developmental timing in Arabidopsis. Cell 138: 750-759.

Xiao, D., J. J. Zhao, X. L. Hou, R. K. Basnet, D. P. Carpio, N. W. Zhang, J. Bucher, K. Lin, F. Cheng, X. W. Wang and G. Bonnema. 2013. The Brassica rapa FLC homolog FLC2 is a key regulator of flowering time, identified through transcriptional co-expression networks. J. Exp. Bot. 64: 4503-4516.

Xu, M., T. Hu, J. Zhao, M. Y. Park, K. W. Earley, G. Wu, L. Yang and R. S. Poething. 2016. Developmental functions of miR156-regulated SQUAMOSA PROMOTER BINDING PROTEIN-LIKE (SPL) genes in Arabidopsis thaliana. PLoS Genet. 12: e1006263.

Zhang, X., L. Meng, B. Liu, Y. Hu, F. Cheng, J. Liang, M. G. M. Aarts, X. Wang and J. Wu. 2016. A transposon insertion in FLOWERING LOCUS $T$ is associated with delayed flowering in Brassica rapa. Plant Sci. 241: 211-220.

Zhao, J., V. Kulkarni, N. Liu, D. P. Del Carpio, J. Bucher and G. Bonnema. 2010. BrFLC2 (FLOWERING LOCUS C) as a candidate gene for a vernalization response QTL in Brassica rapa. J. Exp. Bot. 61: 1817-1825. 